\title{
Bioanalysis
}

\section{Co-medication and interference testing in bioanalysis: a European Bioanalysis Forum recommendation}

Interference testing of co-medication in bioanalytical method validation has become an area of debate in view of the increased specificity offered by current state-ofthe-art technology in both LC-MS/MS and ligand-binding assay platforms. In view of this, and considering the extensive experience within the European Bioanalysis Forum member companies, we evaluated the impact of co-medication on the performance of hundreds of bioanalytical methods with the aim of providing a science-based recommendation on how to evaluate and document potential interference from co-medication on the PK parameters in clinical studies in patients and volunteers.

First draft submitted: 3 June 2016; Accepted for publication: 27 June 2016; Published online: 15 July

Keywords: co-medication $\bullet$ interference testing $\bullet$ method validation

Although selectivity is broadly accepted as a key validation parameter for bioanalytical method validation, the assessment of interference of co-medication on the measured drug and/or metabolite concentrations is challenging and has become an area of debate, both on how to examine the potential interference and to which extent. Over the last couple of years, assessment of interference of co-medication has been discussed within the European Bioanalysis Forum (EBF). In this paper we summarize these discussions and propose a recommendation how to evaluate and document the potential interference of co-medication during assay development, validation and application.

\section{Background}

Bioanalytical method development, validation and the application of the methods to study samples are well defined and conducted according to regulatory guidance. In all the regulatory documents on Bioanalytical Method Validation [1-6] there is guidance on the need to evaluate the possible impact of comedication on the performance of the assay.
The wording varies, but the requirements are consistent as illustrated by the paragraphs in the US FDA [1] or EMA Guidance [2]:

- US-FDA 2001: “...each blank sample should be tested for interference, and selectivity should be ensured at the lower limit of quantification (LLOQ). Potential interfering substances in a biological matrix include endogenous matrix components, metabolites, decomposition products, and in the actual study, concomitant medication and other exogenous xenobiotics. If the method is intended to quantify more than one analyte, each analyte should be tested to ensure that there is no interference."

- EMA 2012 requests to consider the potential impact of interferences on the drug assay: "....It may also be necessary to investigate the extent of any interference caused by metabolites of the drug(s), interference from degradation products formed during sample preparation, and interference from possible co-administered
Marcel de Zwart ${ }^{\ddagger}, 1$, Berthold Lausecker2 ${ }^{2}$, Susanne Globig ${ }^{3}$, Daniel Neddermann ${ }^{4}$, Bruno Le Bras 5 , Alberto Guenzi ${ }^{\ddagger}, 6$, Stephen White', Marianne Scheel-Fjording ${ }^{8}$ \& Philip Timmerman $*, 9$

'PRA Health Sciences, Assen, The Netherlands

${ }^{2}$ CRS Clinical Research Services, Grünstadt, Germany

${ }^{3}$ Actelion Pharmaceuticals Ltd, Allschwil, Switzerland

${ }^{4}$ Novartis Pharma AG, Basel, Switzerland ${ }^{5}$ Servier, Orléans, France

${ }^{6} \mathrm{~F}$. Hoffmann-La Roche, Basel,

Switzerland

${ }^{7}$ GlaxoSmithKline, Ware, UK ${ }^{8}$ Novo Nordisk A/S, Måløv, Denmark 'Janssen R\&D, Turnhoutseweg, 30 B-2340, Beerse, Belgium *Author for correspondence: ptimmerm@its.jnj.com "Retired 
medications. Co-medications normally used in the subject population studied which may potentially interfere should be taken into account at the stage of method validation, or on a study specific and compound specific base."

In practice, the bioanalytical community encountered difficulties to define efficient, consistent and scientific best practices to address co-medication testing during either bioanalytical method validation or application. As a consequence, the EBF felt this issue important and worked on a recommendation to help the bioanalytical community to comply with the regulatory and scientific requirements considering the extensive experience of our member companies, scientific best practices and the being mindful of the resources required on if, how and when co-medication should be tested for interference.

\section{EBF surveys \& discussions}

Discussions on interference testing from co-medication were initiated in EBF discussions in 2012, mainly around the practicality of testing. Main challenges we considered were:

- How and when can the bioanalyst get information about the nature of unscheduled co-medications used in the study?

- How to assess the impact on the study, especially in those (not uncommon) cases when the information on the co-medication only becomes available to the bioanalyst when sample analysis is already complete?

- Is there value in testing in silico instead of performing wet laboratory experiments?

- How to test the multiple drugs and variable doses administered on patients in, for example, cancer therapy, HIV trials or trials in hepatic and renal impaired populations?

- In addition to testing the specificity of the comedication, how to test the potential interference of metabolites of the co-medication?

To ensure we all understood the challenge in the same way during our internal EBF discussions, it was important to align the bioanalytical community and refine the semantics with respect to co-medication. The terminology 'co-medication' was assigned to all medication other than the investigational product administered during a clinical trial. Most common cases are co-medication in relation to drug-drug interaction studies, multiple drug administrations and fixed-dose drug combinations as well as all kinds of drugs used to treat side effects on an individual basis or ongoing therapies of patients in Phase III trials. For practical reasons clarified later in the article, it was important to distinguish between scheduled and unscheduled co-medication:

- Scheduled co-medication: all co-administered drugs for which dose, time and route of administration are defined in the clinical study protocol. Scheduled comedication may be administered to either the entire study population or to predefined sub-groups of the study population.

- Unscheduled co-medication: all drugs which according to the clinical study protocol are allowed to treat side effects and which are applied to single subjects without having a predefined dose, time and route of administration.

The initial discussion helped to prepare two surveys in which the EBF member companies were asked to share their current practices and experience with comedication selectivity testing. Both surveys were set up in a way to separately evaluate the procedures applied in the chromatography-based assay and in the ligand-bind assay (LBA) areas.

The results from the surveys showed that testing for scheduled co-medication was done extensively for LC-MS/MS methods and less for LBA assays. In both cases, regulatory compliance was mentioned as the rationale for performing the experiments. Testing for unscheduled co-medication was performed significantly less, likely related to the challenge of being able to get hold of the information.

In more detail, Survey $\mathbf{1}$ focused on current practices regarding frequency of co-medication assay validation testing and the procedures applied to assess the impact of co-medication on the drug assay. In total, $44 \mathrm{com}$ panies responded, 30 companies reported back on their practices related to LC-MS/MS-based methods (20 Pharma, ten CRO) and 14 with LBA (eight Pharma, six CRO).

The EBF member companies were also asked how they perform the selectivity assessment when testing the interference of the co-administration.

\section{For LC-MS/MS methods}

- The majority of the responders tested for scheduled co-medication (78\%) and a minority tested for unscheduled co-medication (22\%).

- Interference testing was performed in accordance to a bioanalytical study plan $(73 \%)$ or following a process described in a standard operating procedure $(37 \%)$. 
- The most commonly used process is to spike QCsamples (typically the low QC samples) with a predefined concentration of the co-mediation (typically at the nominal concentration of the highest calibration point).

\section{For LBA methods}

- About half of the responders tested for scheduled co-medication $(54 \%)$. None of the responders test for unscheduled co-medication.

- As for LC-MS/MS assays, the most commonly used process is to spike QC-samples (typically the low QC samples) with a predefined concentration of the co-mediation. Unlike for LC-MS/MS assays, the LBA community typically spikes the co-medication to the low $\mathrm{QC}$ at its anticipated $\mathrm{C}_{\max }$.

Both current state-of-the-art LC/MS/MS and LBA technologies claim to have sufficient specificity to waive unscheduled co-medication testing. As an example, companies do not routinely perform wet laboratory experiments for interference testing of co-medication, building on the argument that LC-MS/MS methods are specific to rule out interference. In particular, when a stable labeled isotope is used as internal standard, the majority of these companies argue that MS ensures the specificity of the assay and hence challenged the need of interference testing in the laboratory.

Another argument relates to the impact of potential interference, which is likely to be either minimal or easy to detect. Because in most instances, the unscheduled co-medication is administered to one subject or patient, the unlikely event of a potential analytical problem will be picked up as part of PK evaluations and, as mentioned, will be limited to one or a few samples only.

Survey 2 probed on the frequency of interference testing and the results of these experiments. A total of 39 companies responded, 29 for LC-MS/MS methods (19 Pharma, ten CRO) and ten for LBA methods (five Pharma, five CRO).

For LC-MS/MS methods, the survey reports back on 389 studies (272 for scheduled co-mediation testing, 117 for unscheduled co-medication testing). In our evaluation, we didn't differentiate between methods using of a stable isotope labeled and a structural analogue internal standard. In none of the studies an interference of the co-medication was observed.

The survey did not probe our members if they are performing in silico testing (e.g., MW comparison, $\mathrm{pKa} / \log \mathrm{P}$ evaluation) for those compounds where no wet laboratory interference experiment was performed.

For LBA methods, the survey reports back on Interference of scheduled co-administered drugs in 36 stud- ies. No company tested unscheduled co-administered drugs. In one case, interference was observed which was resolved through changing the assay format and by addition of a specific assay blocking reagent.

\section{Discussion}

The survey data clearly show that the selectivity of the assays developed using modern LC-MS/MS and LBA technology is able to prevent interference from co-medication. Only in one case a member reported interference of co-medication of the $36 \mathrm{LBA}$ assays. In none of the almost 400 LC-MS/MS assays an issue was reported. Similarly, a survey-based paper from the global CRO consortium (GCC), on the potential impact of co-medication on testing drug stability in matrix [10] could not find, within their available dataset, any evidence of impact due to co-medication. It should be noted that we should not summarize the data from GCC and EBF surveys, since both may partially contain data from the same assays as part of dual membership of some EBF CRO member companies in GCC. Nevertheless, data show a similar trend. Ultimately, this does not exclude that there may be circumstances where co-medication and drug are so similar to each other or co-medication might change matrix to such a degree that interference testing is warranted. Typical examples for this in LC-MS/MS-based methods are isobaric compounds.

Evaluation of the data from both EBF surveys, and considering the additional data from the GCC publication, supports the EBF team to propose a recommendation for industry on how to manage the testing of co-medication. Our recommendation does not have the purpose of challenging the respective guidance or guideline documents on this issue, since these guidance documents should consider all potential technologies used in bioanalytical testing. EBF does agree with the guidance provided by regulators and elaborates, based on these documents, further details.

Considering the fact that the vast majority of the assays used in modern drug development are either LC-MS/MS or LBA based, both having an ultimate inherent specificity, we should be open to appreciate the fact that modern technologies have the power to prevent interference of co-medication in most instances. Therefore, EBF proposes a tiered approach to address this issue for LC-MS/MS-based assays and LBA-based assays.

\section{EBF recommendation}

\section{Recommendation for LC-MS/MS assays}

Scheduled co-medication

Because scheduled co-medication is more related to Phase I trials or well documented patient trials, any 


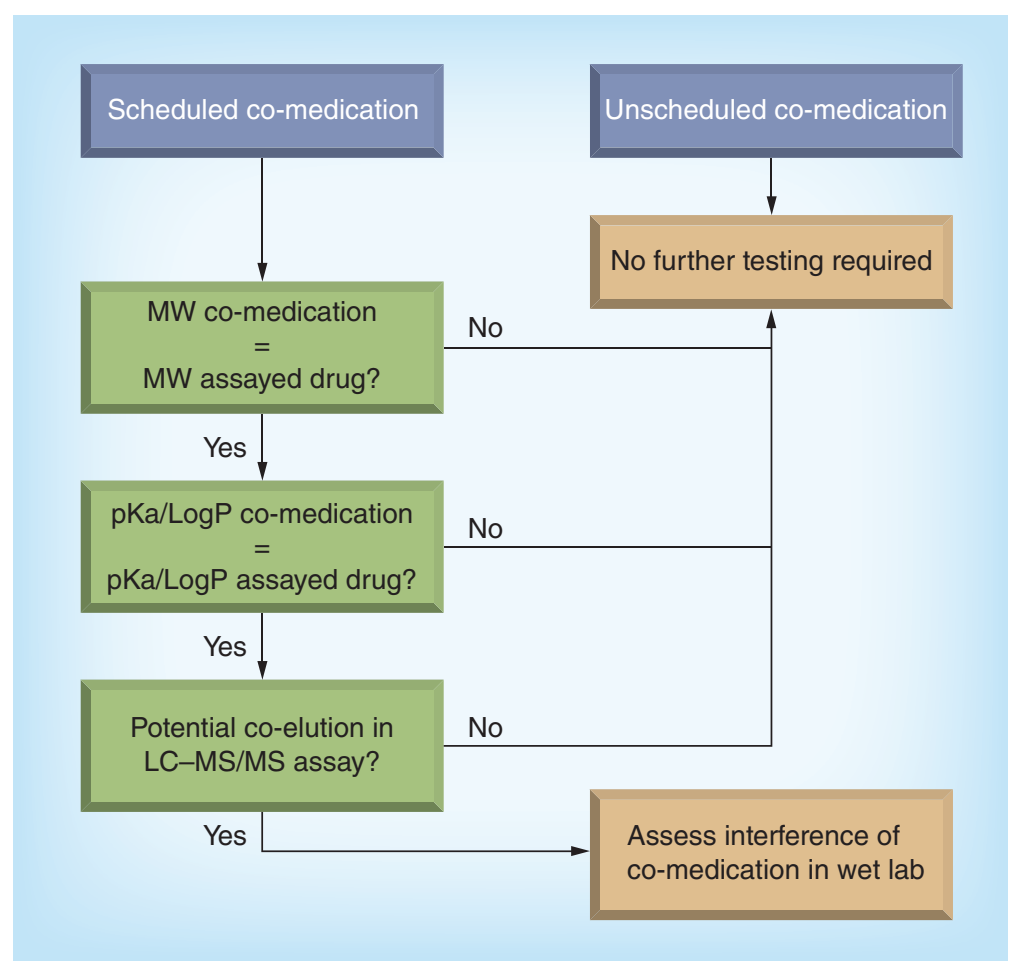

Figure 1. Recommendation for scheduled and unscheduled co-medication testing for LC-MS/MS-based assays.

undue interference may be affecting entire study population or predefined sub-groups of the study population. Hence the following scheme is proposed to prevent undue interference.

- If the MW of the co-medication is different from the assayed drug, the likelihood of MS-based interference of the co-medication is not anticipated and no further testing is needed.

- If the MW of the co-medication is equal to the assayed drug (isobaric drugs), also identify the $\mathrm{pKa}$ and/or $\log \mathrm{P}$ and assess the potential for chromatographic interference.

- In case co-elution is not anticipated under the analytical conditions, the likelihood of LC or MS-based interference of the co-medication is not anticipated and no further testing is needed.

- In case co-elution is anticipated under the analytical conditions of the assay, we recommend assessing the potential interference of the co-medication in the wet laboratory during method development. We suggest spiking the anticipated $\mathrm{C}_{\text {max }}$ concentration of the co-medication to the low QC level of the assayed drug and compare the back-calculated concentrations of the low QC samples with or without addition of the co-medication using regulated
BA acceptance criteria (15\% on accuracy). If there is interference of the co-medication we would recommend to adapt the method and document the lack of interference in method validation.

- We would not recommend the need for a CoA for the tested co-medication. Proof of identity should be documented in the raw data, but any certification would not need to be included in the validation report.

\section{Unscheduled co-medication}

Because unscheduled co-medication is mostly related to Phase III patient trials with sparse sampling, the availability of a full listing of unscheduled co-medication is difficult or even impossible to retrieve. At the same time, an unscheduled co-medication is likely to be a single event in a very limited number of patients. Hence, any undue interference will be affecting single patients or samples and will not have an effect on the study results nor population PK. Therefore the following scheme is proposed to prevent undue effects:

- In view of the low likelihood of interference as shown by the survey data (only one interference for almost 400 investigated assays), and the difficulties (both logistically and from patient confidentiality perspective) to obtain a list of unscheduled co-medication, no testing is recommended.

A schematic overview is shown in Figure 1.

\section{Recommendation for LBA}

Because LBAs are developed using specific antibodies for the dosed drug the following tiered approach can be applied:

- If co-medication is structurally different for the dosed drug, the likelihood of co-medication interference is not anticipated as the antibodies would not recognize the co-medicated compound, and the testing stops here.

- If co-medication is structurally the same as for the dosed drug, and the antibodies used in the LBA assay have different epitope to the drug and comedication, the likelihood of co-medication interference is not anticipated, and the testing stops here.

- If co-medication is structurally the same as for the dosed drug, and the antibodies used in the LBA assay have same epitope to the drug and co-medication, the likelihood of co-medication interference is anticipated and we recommend assessing the potential interference of the co-medication 
in the laboratory. We suggest spiking the anticipated $\mathrm{C}_{\max }$ concentration of the co-medication to the low QC level of the assayed drug and compare the back-calculated concentrations of the low QC samples with or without addition of the comedication using regulated BA acceptance criteria ( $20 \%$ on accuracy). If there is interference of the co-medication we would recommend to use other antibodies or a special blocking reagent.

- As for LC-MS/MS-based assays, we would not recommend the need for a CoA for the tested co-medication. Proof of identity should be documented in the raw data, but any certification would not need to be included in the validation report.

\section{Unscheduled co-medication}

- No specific recommendation.

A schematic overview is shown in Figure 2.

\section{Conclusion}

This article provides feedback from the past and ongoing discussions including survey data within EBF community on how and when to deal with the challenges related to interference testing of co-medication during method development and validation of LC-MS/MS and LBA assays. Survey data on past experience show no instances where co-medication interfered with the LC-MS/MS assays of the investigational drug for both scheduled and unscheduled co-medication (data from 389 observations). For LBA-based assays, the survey data identified only one out of 36 assays where interference was reported. Considering these data and the developments in technology for both LC-MS/MS and LBA assays, EBF proposes a recommendation for documenting potential interference by co-medication for both assay types. The recommendation is based on a priori set criteria, focusses on best scientific practices and considers optimal use of resources of the bioanalytical laboratory. As for previous recommendations, EBF intends to stimulate a harmonized and adequate application of the Guidance principles while recognizing scientific parameters of the compounds and assays.

\section{References}

1 U.S. Department of Health and Human Services, Food and Drug Administration, Center for Drug Evaluation and Research (CDER), Center for Veterinary Medicine (CVM). Guidance for Industry, Bioanalytical Method Validation (2001).

www.fda.gov/downloads/Drugs/Guidance/ucm070107.pdf

2 Viswanathan CT, Bansal S, Booth B et al. Workshop/ conference report - quantitative bioanalytical methods validation and implementation: best practices for

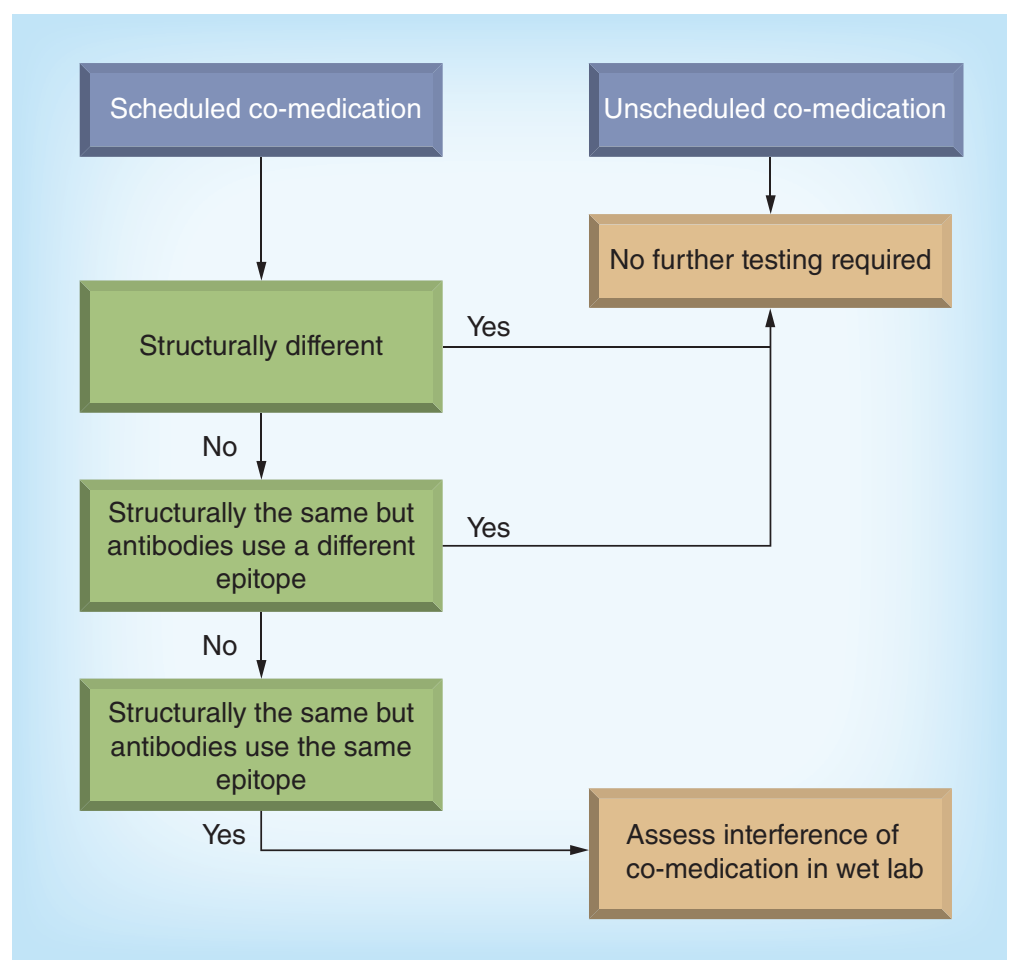

Figure 2. Recommendation for scheduled and unscheduled co-medication testing for ligand-binding assays.

Our recommendation is not applicable for other assay formats than mentioned in the article.

\section{Acknowledgements}

The authors would like to thank all members of the EBF for their participation in the surveys and the discussions on this topic.

\section{Financial \& competing interests disclosure}

The authors have no relevant affiliations or financial involvement with any organization or entity with a financial interest in or financial conflict with the subject matter or materials discussed in the manuscript. This includes employment, consultancies, honoraria, stock ownership or options, expert testimony, grants or patents received or pending, or royalties.

No writing assistance was utilized in the production of this manuscript.

chromatographic and ligand binding assays. AAPS J. 9(1), E30-E42 (2007).

3 Agência Nacional de Vigilância Sanitária (ANVISA), RE 899/2003, May 2003.

www.anvisa.gov.br/medicamentos/RE_899_validacao.pdf

4 European Medicines Agency (EMA), Committee for Medicinal Products for Human Use (CHMP). Guideline on bioanalytical method validation. EMEA/CHMP/ EWP/192217/2009, 21 July 2011.

www.ema.europa.eu 
5 Agência Nacional de Vigilância Sanitária (ANVISA), RDC 27/2012, May 2012.

http://bvsms.saude.gov.br

6 CFDA Guildeline.

http://knex2014.europeanbioanalysisforum.eu

7 Ministry of Health and Welfare (MHLW). Guideline on Bioanalytical Method Validation in Pharmaceutical Development, Japan, September 2013. www.nihs.go.jp/drug/BMV/BMV_draft_130415_E.pdf

8 U.S. Department of Health and Human Services, Food and Drug Administration, Center for Drug Evaluation and Research (CDER), Center for Veterinary Medicine (CVM).
Guidance for Industry, Bioanalytical Method Validation. Draft, September 2013.

www.fda.gov

9 DeSilva B, Garofolo F, Rocci M et al. White paper on recent issues in bioanalysis and alignment of multiple guidelines. Bioanalysis 4(18), 2213-2226 (2012).

10 Lowes S, Boterman M, Doig M et al. Recommendations on bioanalytical method stability implications of coadministered and co-formulated drugs by Global CRO Council for Bioanalysis (GCC). Bioanalysis 4(17), 2117-2126 (2012) 